\title{
BEAM DYNAMICS OF NON-EQUIPARTITIONED BEAMS IN THE CASE OF THE SPL PROJECT AT CERN
}

\author{
Frank Gerigk, CERN, Geneva, Switzerland \\ Ingo Hofmann, GSI Darmstadt, Germany
}

\begin{abstract}
The SPL [1] working group at CERN is studying a 2.2 $\mathrm{GeV} \mathrm{H}^{-}$linac, which recuperates a large amount of $\mathrm{RF}$ hardware from the now decommissioned LEP at CERN. During the ongoing design effort for an optimized layout, it was found that in some cases non-equipartitioned beams tend to exchange energy between the longitudinal and the transverse planes. Strict energy equipartition, however, imposes tight restrictions on such a high energy linac and often contradicts the goal of cost effective design. On the other hand, stability charts derived from 2D Vlasov analysis suggest the existence of stable non-equipartitioned equilibria in certain regions of parameter space. Due to the low bunch current $(22 \mathrm{~mA})$ in the SPL, these regions are large enough to ensure stable machine operation for nonequipartitioned beams. Systematic multiparticle simulations with IMPACT [2] are used to apply the stability charts to the beam dynamics design of a realistic high energy linac. Using the example of the SPL, it is shown that designs with stable non-equipartitioned bunches are feasible, and how these designs react to mismatched input beams.
\end{abstract}

\section{INTRODUCTION}

Since the emittance exchange in unstable areas of the stability charts developed by Hofmann [3], [4] has already been demonstrated for idealized cases, the goal of this study is to establish the validity of the charts for a realistic linac set-up, using a periodic focusing structure and 3D bunched beams. For this purpose the first two superconducting sections of the SPL [5] were chosen to benchmark different areas in the parameter space of the charts. These sections include a transition with a change of period length, as well as phase slippage in the multicell cavities, a phenomenon occurring in all present designs of superconducting linacs. Both sections consist of 4-cell cavities, designed for the particle velocities of $\beta=0.52$ and $\beta=0.7$.

\section{SIMULATIONS}

All simulations use approximately twice the design current (40mA) of the actual layout (Table 1) and start with an initial 6D Waterbag distribution. Four realistic cases were simulated to explore the parameter space on the stability chart, which was calculated for the SPL emittance ratio of $\varepsilon_{l} / \varepsilon_{t} \approx 2$ (Fig.1). The chart indicates regions (in grey) where third and fourth order modes of collective space charge density oscillations are expected to cause emittance transfer. It is noted that the dangerous regions
Table 1: Main layout parameters of simulated structure

\begin{tabular}{|l|c|c|}
\hline Particles & $\mathrm{H}^{-}$ & \\
\hline Injection energy & 120 & $\mathrm{MeV}$ \\
\hline $\begin{array}{l}\text { Transition energy between } \\
\beta=0.52 \text { and } \beta=0.7\end{array}$ & 236 & $\mathrm{MeV}$ \\
\hline Output energy & 383 & $\mathrm{MeV}$ \\
\hline No. of focusing periods & 22 & \\
\hline RF frequency & 352.2 & $\mathrm{MHz}$ \\
\hline Max. bunch current & $22(40)^{*}$ & $\mathrm{~mA}$ \\
\hline$\varepsilon_{\text {t,r.m.s., norm. }}$ & 0.4 & $\pi \mathrm{mm} \mathrm{mrad}$ \\
\hline$\varepsilon_{l, \text { r.m.s. }}$ & 0.755 & $\pi \mathrm{mm} \mathrm{mrad}$ \\
\hline
\end{tabular}

have a resonance structure, with a predominant resonance around tune ratio 1 , which is caused by a fourth order mode (corresponding to a relationship $2 \sigma_{l}-2 \sigma_{t} \approx 0$ in a single particle picture).

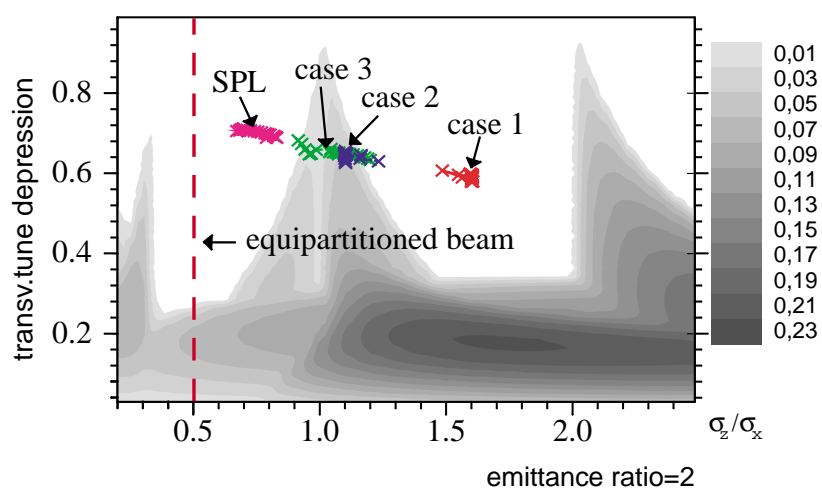

Figure 1: Stability chart for SPL nominal emittance ratio with set-ups for different simulations $(120 \mathrm{MeV}$ - $383 \mathrm{MeV}$ ). Regions are shown, where theory predicts emittance exchange (grey scales indicate theoretical growth rates of resonances in terms of transverse betatron periods; stability occurs in white regions).

All four cases use the same longitudinal settings, but different quadrupole adjustments. The phase advance in all three planes always remains below $90^{\circ}$, with maximum values for the SPL case $\left(\sigma_{t 0}<80^{\circ}\right)$ and minimum values for case $1\left(\sigma_{t 0}>36^{\circ}\right)$.

A modified version of the envelope code FIX3D [6] is used to fit the quadrupole settings for the desired ratios of the matched full current tunes. Cases 1 and 2 are fitted for constant ratios of 1.6 and 1.1, while case 3 and the SPL scan along a line in the chart. Due to the matching between 
the sections, there are three points per case outside these ratios. Table 2 lists the main characteristics of the four cases and Fig. 2 depicts the simulation results for a matched input beam.

Table 2: Boundaries of the four cases

\begin{tabular}{|l|c|c|c|c|}
\hline & case 1 & case 2 & case 3 & SPL \\
\hline$\sigma_{t 0}$ & $37^{\circ}-52^{o}$ & $45^{\circ}-65^{\circ}$ & $45^{\circ}-62^{\circ}$ & $55^{\circ}-79^{\circ}$ \\
\hline$\sigma_{l 0}$ & $45^{\circ}-62^{\circ}$ & $44^{\circ}-62^{\circ}$ & $42^{\circ}-62^{\circ}$ & $42^{\circ}-62^{\circ}$ \\
\hline$\sigma_{t} / \sigma_{t 0}$ & $.58-.61$ & $.64-.67$ & $.63-.68$ & $.68-.72$ \\
\hline$\sigma_{l} / \sigma_{l 0}$ & $.77-.78$ & $.73-.75$ & $.72-.75$ & $.68-.72$ \\
\hline anisotr.* & $2.7-3.1$ & $2.1-2.4$ & $2.3-1.7$ & $1.6-1.3$ \\
\hline \multicolumn{6}{|c}{$*$ anisotropy defined as $:\left(\sigma_{l} \cdot \varepsilon_{l}\right) /\left(\sigma_{t} \cdot \varepsilon_{t}\right)$}
\end{tabular}

case 1

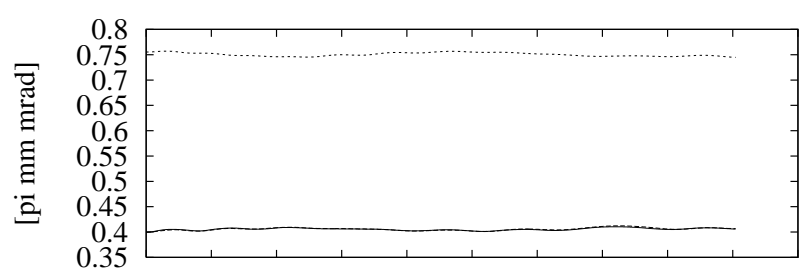

case 2

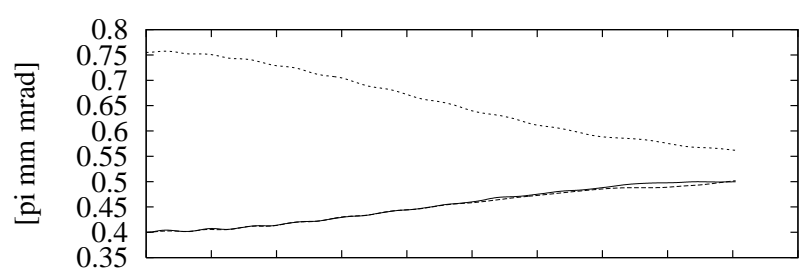

case 3

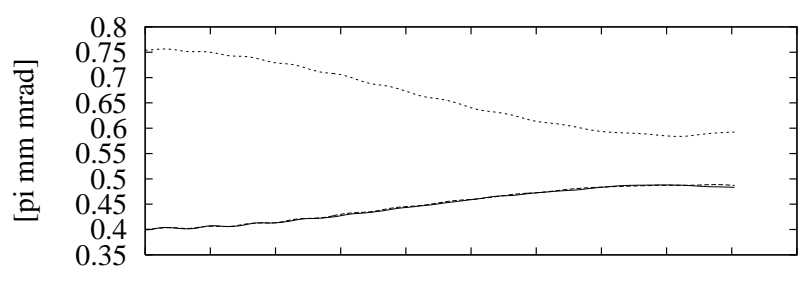

SPL

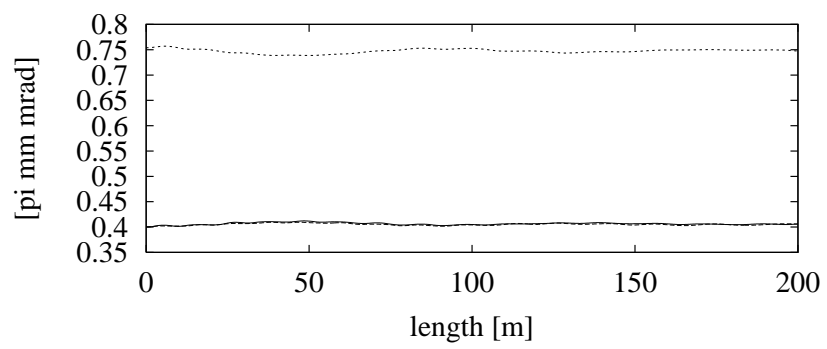

Figure 2: R.m.s. emittance evolution (upper curves: longitudinal, lower curves: transverse)

Case 1, which has the highest anisotropy ratio (3.1) between the longitudinal and the transverse plane, and the SPL show a practically constant emittance evolution (see also Table 2) while in cases 2 and 3 a clear energy exchange between the longitudinal and the transverse plane can be observed. This exchange involves: 1.) a rise of the matched tunes in the transverse plane and a decrease in the longitudinal plane, meaning that the beam moves out of the unstable region towards equipartitioning; 2 .) a reduced emittance ratio and therefore a changing chart topology, resulting in a shrinking size of the unstable area and again a move towards equipartitioning; and

3.) due to the changing r.m.s. emittances the beam becomes mismatched. The changing tune and emittance ratios are not included in Fig.1.

Since the coupling resonances are induced by space charge one expects that the outermost particles react differently to the core particles. Simulations with $10^{6}$ particles

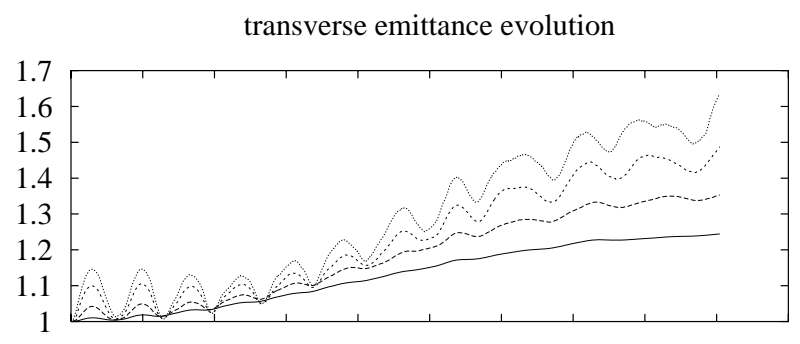

longitudinal emittance evolution

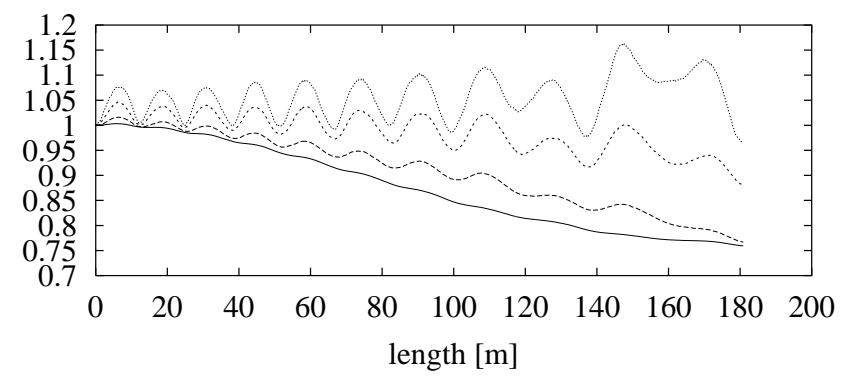

Figure 3: Evolution of fractional normalized emittances for case 2 in ascending order: r.m.s., 99\%, 99.9\%, and $99.99 \%$

on a $128^{3}$ space charge grid show that in case of r.m.s. emittance reduction (here in the longitudinal plane, lower part of Fig. 3) the outer $0.1 \%$ fraction of the particles are hardly influenced by the changing core distribution, whereas the 99\% emittance is still considerably reduced. In case of emittance growth (here in the transverse plane, upper part of Fig. 3) the outer particles first show a delayed reaction to the expanding core but then experience twice the growth of the r.m.s. emittance. These results indicate a clear migration of particles into a diffused beam halo due to the emittance exchange via space charge resonances, a process that should at all costs be avoided in the design of high intensity linacs. However, a fast transition of an unstable area might be possible with only moderate emittance degradation.

Table 3: Relative emittance growth for matched input beams at the end of the simulated linac $(383 \mathrm{MeV})$

\begin{tabular}{|l|c|c|c|c|}
\hline case & $\varepsilon_{t, r . m . s .}$ & $\varepsilon_{l, r . m . s .}$ & $\varepsilon_{t, 99.99 \%}$ & $\varepsilon_{l, 99.99 \%}$ \\
\hline SPL & $1.01 / 1.01$ & .994 & $1.16 / 1.20$ & 1.11 \\
1 & $1.02 / 1.02$ & .986 & $1.23 / 1.20$ & 1.08 \\
2 & $1.24 / 1.23$ & .759 & $1.63 / 1.62$ & 1.13 \\
3 & $1.20 / 1.20$ & .793 & $1.45 / 1.43$ & 1.08 \\
\hline
\end{tabular}




\section{MISMATCH STUDY}

To study the effect of mismatched input beams for different lattices we follow the approach suggested in [7]. We excite the three envelope modes of mismatched bunched beams (related to the Quadrupolar-, High-frequency, and Low-frequency mode, though we are not rigorously exciting the eigenmodes) so that the largest oscillation has a relative amplitude of 1.3 corresponding to a $30 \%$ mismatch. Fig. 4 shows the ratio between the mismatched and

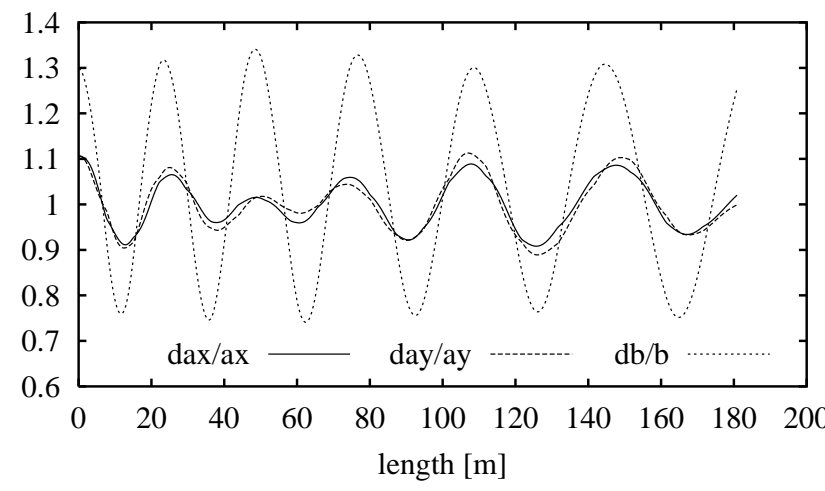

Figure 4: Ratio between the mismatched and matched r.m.s. radii in case of a $30 \%$ High-frequency mode excitation for case 1

the matched r.m.s. radii for case 1. Here the longitudinal oscillation has the largest amplitude and is set to 1.3. The anti-phased transverse oscillations have an amplitude of 1.1 .

Table 4: Relative emittance growth for mismatched input beams $(120 \mathrm{MeV}-383 \mathrm{MeV})$

\begin{tabular}{|l|c|c|c|c|}
\hline case/mode & $\varepsilon_{t, r . m . s .}$ & $\varepsilon_{l, r . m . s .}$ & $\varepsilon_{t, 99.99 \%}$ & $\varepsilon_{l, 99.99 \%}$ \\
\hline SPL/Q & $1.11 / 1.09$ & .997 & $4.68 / 3.97$ & 1.10 \\
1/Q & $1.08 / 1.04$ & .988 & $3.85 / 2.38$ & 1.09 \\
2/Q & $1.27 / 1.27$ & .799 & $3.51 / 3.30$ & .992 \\
3/Q & $1.23 / 1.25$ & .837 & $3.67 / 3.43$ & .993 \\
\hline SPL/L & $1.01 / 1.01$ & 1.01 & $1.21 / 1.18$ & 2.58 \\
1/L & $1.02 / 1.02$ & .990 & $1.38 / 1.23$ & 1.15 \\
2/L & $1.24 / 1.21$ & .835 & $1.94 / 1.83$ & 3.26 \\
3/L & $1.19 / 1.19$ & .894 & $2.12 / 1.86$ & 3.46 \\
\hline SPL/H & $1.03 / 1.02$ & .994 & $1.92 / 1.28$ & 1.10 \\
1/H & $1.01 / 1.01$ & .993 & $1.20 / 1.18$ & 1.22 \\
2/H & $1.26 / 1.20$ & .787 & $2.54 / 1.47$ & 1.09 \\
3/H & $1.20 / 1.20$ & .805 & $1.45 / 1.42$ & 1.16 \\
\hline
\end{tabular}

Case 1 can be considered as an alternative layout to the SPL, since it is the only one that does not exchange emittances in the matched case. Comparing the sensitivity to mismatch mode excitation, the SPL emittance growth rates seem to be slightly higher than for case 1 . However, going to higher energies, case 1 would have to cross the fourth order instability due to the decreasing longitudinal phase advance. Since the transverse focusing for case 1 is weaker than for the SPL, the transverse matched beam radius is smaller in the SPL lattice (this still applies for mismatched input beams). Therefore the SPL lattice was chosen as the reference layout for the project. Cases 2 and 3 still show a clear r.m.s. emittance exchange, indicating that the process of energy exchange between the planes is not sensitive to mismatch. All cases show distinctly increased halo production for mismatched input beams.

\section{CONCLUSIONS}

A good agreement has been established between the prediction of unstable areas derived from 2D Vlasov analysis, and fully 3D PIC simulations of realistic designs. Beams in unstable areas of the theoretical Stability Charts show distinct emittance exchange in spite of the relatively low tune depression of $\approx 0.7$. Due to the exchange, these beams move towards stable areas in the charts in the direction of equipartitioning. It has also been demonstrated that the non-equipartitioned equilibria predicted by the charts exist (see also [8], [9]) and that, in case of the SPL layout, these equilibria do not show distinctly higher or lower sensitivity to mismatched input beams. The energy exchange for mismatched beams is of the same order as for matched beams, although the values get blurred by an overall tendency for emittance growth and halo formation. In the design of high intensity linacs, the unstable areas should be avoided due to the development of beam halo during energy exchange. However, the option of a fast transition of unstable areas should be a subject for further studies.

\section{ACKNOWLEDGEMENTS}

This research used resources of the National Energy Research Scientific Computing Center.

\section{REFERENCES}

[1] Ed. M. Vretenar. Conceptual Design of the SPL, a HighPower Superconducting H-Linac at CERN, CERN 2000-012.

[2] J. Qiang; R.D. Ryne; S. Habib; V. Decyk. An ObjectOriented Parallel Particle-In-Cell Code for Beam Dynamics Simulation in Linear Accelerators. Journal of Computational Physics, 163:1-18, 2000.

[3] I. Hofmann. Stability of Anisotropic Beams with Space Charge. In Physical Review E 57, 4713, 1998.

[4] I. Hofmann; J. Qiang; R.D. Ryne. Collective Resonance Model of Energy Exchange in 3D Non-Equipartitioned Beams. Phys.Rev.Lett. 86, accepted, 2001.

[5] F. Gerigk; M. Vretenar; R.D. Ryne. this conference.

[6] R.D. Ryne. Finding Matched RMS Envelopes in RF Linacs: A Hamiltionian Approach, LA-UR-95-391. LANL, Los Alamos, NM 87545, 1995.

[7] A. Letchford; K. Bongardt; M. Pabst. Halo Formation of Bunched Beams in Periodic Focusing Systems. In Proc. PAC 1999, page 1767, New York.

[8] K. Bongardt; M. Pabst; A. Letchford. High Intensity Injector Linacs for Spallation Sources. In Proc. LINAC 98, page 339.

[9] J-M. Lagniel; S. Nath. On Energy Equipartition Induced by Space Charge in Bunched Beams. In Proceedings of EPAC 98, page 1118 . 